\title{
Analysis of Volatiles from Fresh and Dry Rhizome, Pseudostem and Leaf of Musa Basjoo
}

\author{
Chenxi Zhu, Feng Xu, Hongmei Wu*, Xiangpei Wang \\ Guiyang University of Chinese Medicine, 50, Nanming District, Guiyang City, Guizhou Province, \\ PR China \\ ${ }^{*}$ Correspondence to: whm0425@126.com
}

\begin{abstract}
Keywords: Musa basjoo Sieb. et Zucc., Volatiles, Fresh and dry, Rhizome, Pseudostem, Leaf Abstract. Background: Musa basjoo Sieb. et Zucc. is widely distributed around the world, used to treat a variety of diseases. Volatile oils as an important component of the Musa basjoo, but it received less attention. In this study, Volatile constituents in both fresh and dry rhizome, pseudostem and leaf of Musa basjoo were studied.

Materials and Methods: a gas chromatography-mass spectrometry (GC-MS) with solid-phase micro extrac-tion (SPME) method was developed for the analysis of fresh and dry rhizome, pseudostem and leaf of Musa basjoo.

Results: 60, 73, 52, 70, 31, and 45 volatile components were indentified from fresh rhizome, dry rhizome, fresh pseudostem, dry pseudostem, fresh leaf and dry leaf, respectively. Only 14 compounds were mutual in the volatiles of six Musa basjoo parts. Comparison among fresh Musa basjoo rhizome, pseudostem and leaf, the mutual compounds were 30, 18, 14 compounds, respectively. The main compounds were Hexadecanal and Hexhanal. 35 mutual compounds were detected in the dry rhizome, pseudostem and leaf samples. 59, 36, 40 compounds were identified as the mutual constituents in the volatiles of dry rhizome, pseudostem and leaf, respectively.

Conclusion: the results showed that the volatiles from fresh and dry rhizome, pseudostem and leaf of Musa basjoo are quite different. And this work was conductive to the development and utilization of Musa basjoo.
\end{abstract}

\section{Introduction}

Cultivated and wild resources Musa basjoo Sieb. et Zucc. are wide distributed in China. The dried rhizome of Musa basjoo is Rhizoma Musae, a herb has been used for centuries in Miao medicine practice for heat-clearing and detoxifying treatments, as well as quenching of thirst and as a diuretic (Drug Administration of Guizhou Province, 2003; Bao, J., 1999). Musa basjoo pseudostem juice is used to treatment of convulsion, epilepsy, high blood pressure headache, otitis media, burns etc., Musa basjoo leaf has heat, diuretic, detoxification active. the two herbs records in the Chinese materia medica (Editorial board of state administration of traditional Chinese medicine for Chinese herbal medicine, 1999). several studies have suggested that Rhizoma Musae has anti-bacteria, anti$\alpha$-glucosidase, anti-inflammatory, anti-diabetic, and analgesic properties (Qian et al. 2010; Wei et al. 2010; Zhang et al. 2010; Qian et al. 2012; Xu et al. 2014; Zhang et al. 2010), another study has shown that the alkaloids of Musa basjoo pseudostem exhibits acute hypotensive effect on dog (Zhu et al. 1989).

Volatile oil is an important component of medicinal plants. Therefore, the research on volatiles is important. The volatile constituents from fresh and dry Rhizoma Musae extracted by steam distillation have been determined by gas chromatography with less identified volatile compounds, accounting for $76.28 \%$ and $81.78 \%$, respectively (Wang et al. 2011). The volatiles of fresh and dry Musa basjoo pseudostem and leaf have not detected. Differences in Musa basjoo rhizome, pseudostem and leaf volatiles also have not compared. So in the paper, six medicinal parts (fresh and dry rhizome, pseudostem and leaf) of Musa basjoo were analysis by gas chromatography-mass spectrometry (GC-MS) with solid-phase micro extraction (SPME). Then, the comparison among the fresh and dry Musa basjoo rhizome, pseudostem and leaf was made. A comparative analysis on volatiles in different parts of Musa basjoo also evaluated. 


\section{Plant Materials}

The Musa basjoo whole plant was collected from Jinsha county, guizhou province, P.R. China, in 2014 and further identified by Dr. X.P. Wang, Department of Pharmacognosy Guiyang College of Traditional Chinese Medicine, P. R. China. All voucher specimens were deposited in the Department of Pharmacognosy, Guiyang College of Traditional Chinese Medicine, P.R. China. The Musa basjoo rhizome, pseudostem and leaf were cut into small pieces, half of the samples were stored in a refrigerator and the temperature was set at $-20{ }^{\circ} \mathrm{C}$, the other samples were placed in a cool dark place (temperatures between $20-30{ }^{\circ} \mathrm{C}$ ) to dry naturally.

\section{Solid-Phase Micro Extraction Procedure}

The accurately weighed fresh and dry rhizome, pseudostem and leaf small pieces $(5.0 \mathrm{~g})$ of $\mathrm{Musa}$ basjoo were placed into 20-mL sample vials from Supelco (Bellefonte, USA), respectively. Then, a 2cm-50/30um DVB-CAR-PDMS Stable Flex fiber (Bellefonte, USA) was used to the headspace above the samples for extracting 30 min under about $90{ }^{\circ} \mathrm{C}$. then the extraction head was removed from sample vials and immediately inserted onto the GC injection port. Desorption of analytes from the fiber coating was performed by heating the fiber in the injection port at $250{ }^{\circ} \mathrm{C}$ for 3 min.

\section{Determination Conditions}

HP6890 GC-5975C MSD (Agilent Technologie s, Palo Alto, CA, U.S.A.) using a ZB-5MSI (5\% Phenyl-95\% DiMethylpolysiloxane) fused silica capillary column $(30 \mathrm{~m} \times 0.25 \mathrm{~mm} \times 0.25 \mu \mathrm{m})$ was used for constituents separation. The samples were injected in the splitless mode at $250{ }^{\circ} \mathrm{C}$. the initial column temperature was kept at $40{ }^{\circ} \mathrm{C}$ for $2 \mathrm{~min}$, then adjusted to $280{ }^{\circ} \mathrm{C}$ at a rate of $4{ }^{\circ} \mathrm{C}$ /min and held for 2 min. High-purity of Helium (99.999\%) was used as the carrier gas at a flow rate of $1.0 \mathrm{ml} / \mathrm{min}$.

The mass spectrometer was fitted with an EI source operated at $70 \mathrm{eV}$, the source temperature was $230{ }^{\circ} \mathrm{C}$, and the interface temperature was $280{ }^{\circ} \mathrm{C}$ with a solvent delay time of $1.5 \mathrm{~min}$. Mass spectra were recorded in the full scan acquisition mode ( $\mathrm{m} / \mathrm{z}$ range $20-450)$. Volatile compounds were identified by comparing the obtained mass spectra of the analytes with those of authentic standards from the NIST2005 library and Wiley275 library.

\section{Results and Discussion}

Volatile of many herbs in China is responsible for their active substances. In order to study Musa basjoo volatile components, the fresh and dry rhizome, pseudostem and leaf of Musa basjoo samples were subjected to gas chromatography-mass spectrometry analysis. The volatiles in the fresh and dry rhizome, pseudostem and leaf of Musa basjoo are presented in the table 1-3 and figure 1-3. 


\section{Figure 1}

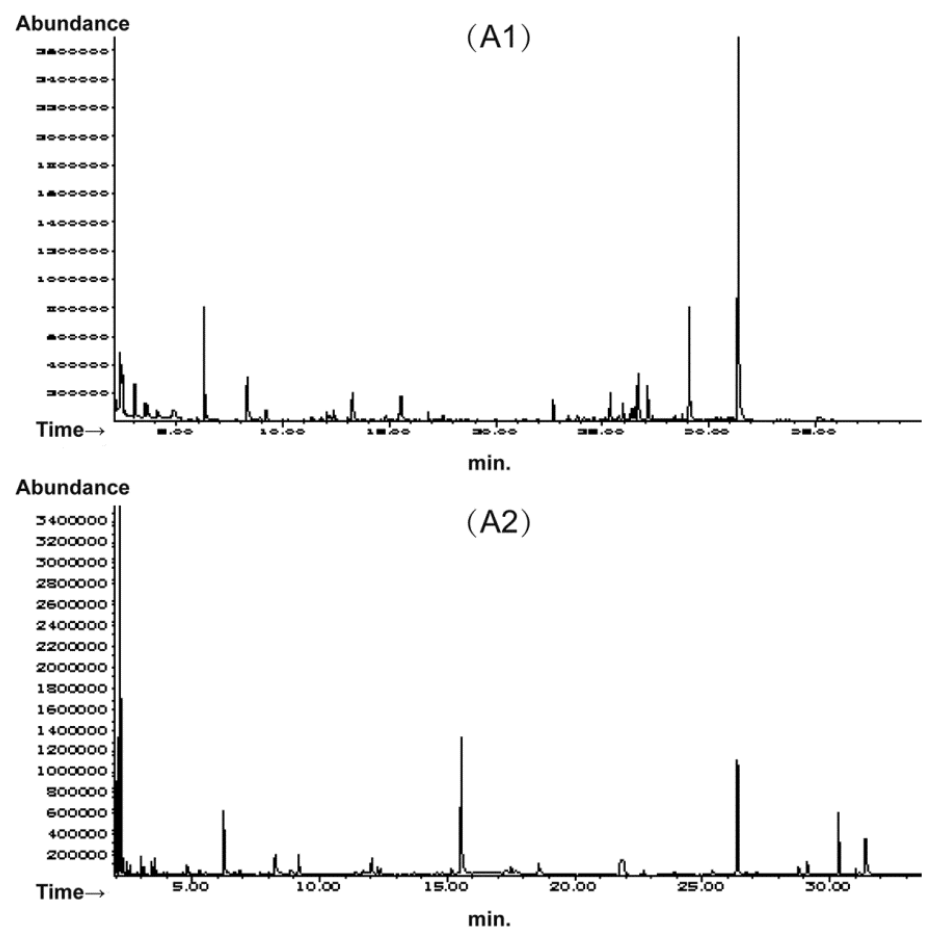

Figure 1. Typical total ion chromatograms of the volatile constituents from fresh (A1) and dry (A2) rhizome of Musa basjoo

\section{Figure 2}
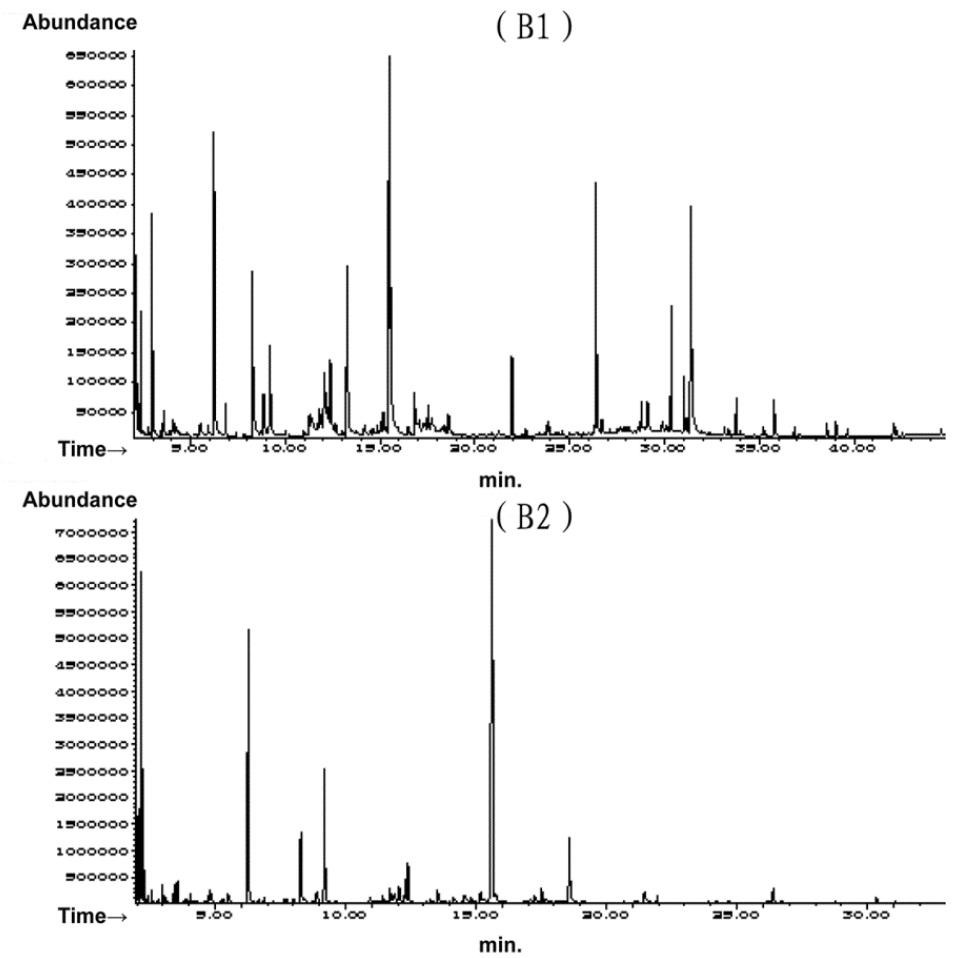

Figure 2. Typical total ion chromatograms of the volatile constituents from fresh (B1) and dry (B2) pseudostem of Musa basjoo 


\section{Figure 3}

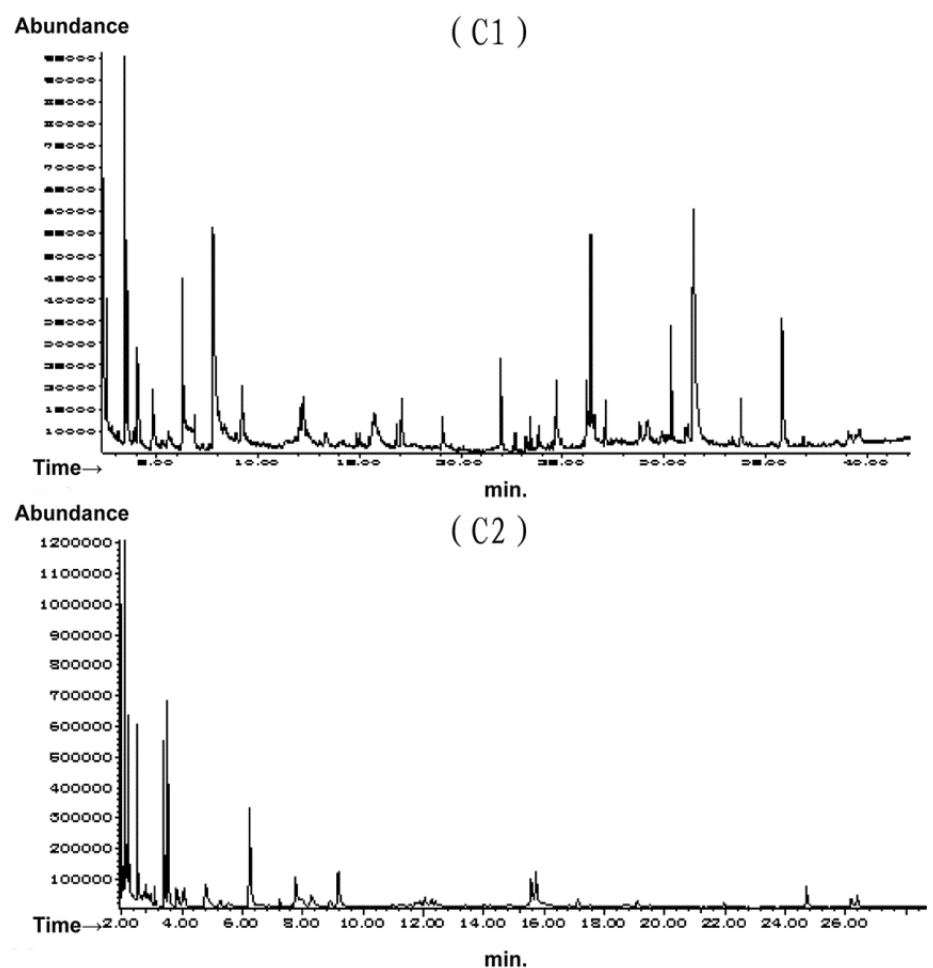

Figure 3. Typical total ion chromatograms of the volatile constituents from fresh $(\mathrm{C} 1)$ and dry (C2) leaf of Musa basjoo

60 compounds were identified from the fresh Musa basjoo rhizome, accounting for the 93.83\%, The main constituents were Hexadecanal (32.3\%), Hexanal (7.56\%),1-Hexanol (5.28\%), Hexadecanol (4.38\%), and Methyl acetate (3.31\%). while, in the dry Musa basjoo rhizome, there were 73 chemical compositions were identified, which comprised $96.33 \%$ of the total volatile fraction (table 1). The content of Nonanal (17.80\%), Ethanol (17.63\%), Hexanal (7.33\%), Ethanal (3.70\%), 1-Hexanol (7.33\%) was higher in the total volatiles. This result did not agreement with the previous studies (Wang et al. 2011). The volatile compounds of Rhizoma Musae may be affected by seasonal, location, sample pretreatment methods, extraction methods, and individuals. And 35 compounds were simultaneously detected in fresh and dry Rhizoma Musae. Alcohols (17.44\%), aldehydes $(56.12 \%)$, alkenes $(10.45 \%)$ were the main compositions in the fresh Rhizoma Musae, but the content of these compositions were reduced in the drying process.

Table 1 Volatiles from fresh and dry rhizome of Musa basjoo

\begin{tabular}{|c|c|c|c|c|c|c|}
\hline \multirow[t]{2}{*}{ No. } & \multirow{2}{*}{$\begin{array}{l}\text { Retentio } \\
\mathrm{n} \text { time } \\
(\mathrm{min})\end{array}$} & \multirow[t]{2}{*}{ Compound name } & \multirow[t]{2}{*}{ Formula } & \multirow{2}{*}{$\begin{array}{l}\text { Molecular } \\
\text { Weight }\end{array}$} & \multicolumn{2}{|c|}{ The percentage (\%) } \\
\hline & & & & & Fresh samples & Dry samples \\
\hline 1 & 2.02 & Ethanal & $\mathrm{C}_{2} \mathrm{H}_{4} \mathrm{O}$ & 44 & - & 4.24 \\
\hline 2 & 2.14 & Ethanol & $\mathrm{C}_{2} \mathrm{H}_{6} \mathrm{O}$ & 46 & - & 17.63 \\
\hline 3 & 2.44 & Methyl acetate & $\mathrm{C}_{3} \mathrm{H}_{6} \mathrm{O}$ & 74 & 3.31 & 0.66 \\
\hline 4 & 2.55 & Isobutanal & $\mathrm{C}_{4} \mathrm{H}_{8} \mathrm{O}$ & 72 & - & 0.65 \\
\hline 5 & 2.59 & Isobutanol & $\mathrm{C}_{4} \mathrm{H}_{8} \mathrm{O}$ & 72 & 0.25 & - \\
\hline 6 & 2.63 & (E)-2-Butanal & $\mathrm{C}_{4} \mathrm{H}_{6} \mathrm{O}$ & 70 & - & 0.05 \\
\hline 7 & 2.83 & 2-Butanone & $\mathrm{C}_{4} \mathrm{H}_{8} \mathrm{O}$ & 72 & - & 0.15 \\
\hline 8 & 3.02 & Ethyl acetate & $\mathrm{C}_{4} \mathrm{H}_{8} \mathrm{O}_{2}$ & 88 & 2.43 & 1.04 \\
\hline 9 & 3.12 & Isobutanol & $\mathrm{C}_{4} \mathrm{H}_{10} \mathrm{O}$ & 74 & - & 0.51 \\
\hline 10 & 3.47 & 3-methyl-Butanal & $\mathrm{C}_{5} \mathrm{H}_{10} \mathrm{O}$ & 86 & 0.81 & 1.04 \\
\hline 11 & 3.59 & 2-methyl-Butanal & $\mathrm{C}_{5} \mathrm{H}_{10} \mathrm{O}$ & 86 & 0.97 & 1.35 \\
\hline 12 & 3.84 & 1-Penten-3-ol & $\mathrm{C}_{5} \mathrm{H}_{10} \mathrm{O}$ & 86 & - & 0.05 \\
\hline
\end{tabular}




\begin{tabular}{|c|c|c|c|c|c|c|}
\hline 13 & 3.88 & Triethylamine & $\mathrm{C}_{6} \mathrm{H}_{15} \mathrm{~N}$ & 101 & - & 0.37 \\
\hline 14 & 4.09 & Pentanal & $\mathrm{C}_{5} \mathrm{H}_{10} \mathrm{O}$ & 86 & 0.59 & 0.29 \\
\hline 15 & 4.12 & 2-ethylfuran & $\mathrm{C}_{6} \mathrm{H}_{8} \mathrm{O}$ & 96 & - & 0.20 \\
\hline 16 & 4.31 & Ethyl propionate & $\mathrm{C}_{5} \mathrm{H}_{10} \mathrm{O}_{2}$ & 102 & - & 0.11 \\
\hline 17 & 4.61 & 2,4,5-trimethyl-1,3-dioxolane & $\mathrm{C}_{6} \mathrm{H}_{12} \mathrm{O}_{2}$ & 116 & - & 0.21 \\
\hline 18 & 4.68 & 1,1-diethoxy-Ethane & $\mathrm{C}_{6} \mathrm{H}_{14} \mathrm{O}_{2}$ & 118 & - & 0.17 \\
\hline 19 & 4.77 & 3-methyl-1-Butanol & $\mathrm{C}_{5} \mathrm{H}_{12} \mathrm{O}$ & 88 & 0.80 & 0.94 \\
\hline 20 & 4.87 & 2-methyl-1-Butanol & $\mathrm{C}_{5} \mathrm{H}_{12} \mathrm{O}$ & 88 & 0.77 & 0.99 \\
\hline 21 & 5.29 & Ethyl isobutyrate & $\mathrm{C}_{6} \mathrm{H}_{12} \mathrm{O}_{2}$ & 116 & - & 0.38 \\
\hline 22 & 5.6 & 1-Pentanol & $\mathrm{C}_{5} \mathrm{H}_{12} \mathrm{O}$ & 88 & 0.27 & 0.30 \\
\hline 23 & 5.96 & Diethyl carbonate & $\mathrm{C}_{5} \mathrm{H}_{10} \mathrm{O}_{3}$ & 118 & 0.26 & - \\
\hline 24 & 6.28 & Hexanal & $\mathrm{C}_{6} \mathrm{H}_{12} \mathrm{O}$ & 100 & 7.56 & 7.33 \\
\hline 25 & 6.66 & Butyl acetate & $\mathrm{C}_{6} \mathrm{H}_{12} \mathrm{O}_{2}$ & 116 & - & 0.33 \\
\hline 26 & 7.25 & 3-methyl-Hexan-2-one & $\mathrm{C}_{6} \mathrm{H}_{10} \mathrm{O}_{2}$ & 114 & - & 0.04 \\
\hline 27 & 7.67 & Ethyl 2-methylbutyrate & $\mathrm{C}_{7} \mathrm{H}_{14} \mathrm{O}_{2}$ & 130 & - & 0.23 \\
\hline 28 & 7.79 & (Z)-3-Hexenal & $\mathrm{C}_{6} \mathrm{H}_{10} \mathrm{O}$ & 98 & 0.23 & 0.30 \\
\hline 29 & 8 & Ethyl benzene & $\mathrm{C}_{8} \mathrm{H}_{10}$ & 106 & - & 0.15 \\
\hline 30 & 8.31 & 1-Hexanol & $\mathrm{C}_{6} \mathrm{H}_{14} \mathrm{O}$ & 102 & 5.28 & 3.70 \\
\hline 31 & 8.45 & Isoamyl acetate & $\mathrm{C}_{7} \mathrm{H}_{14} \mathrm{O}_{2}$ & 130 & - & 0.38 \\
\hline 32 & 8.74 & 5-methyl-3-methylene-5-Hexen-2-one & $\mathrm{C}_{8} \mathrm{H}_{12} \mathrm{O}$ & 124 & - & 0.09 \\
\hline 33 & 8.89 & Styrene & $\mathrm{C}_{8} \mathrm{H}_{8}$ & 104 & - & 0.49 \\
\hline 34 & 8.9 & 2-Heptanone & $\mathrm{C}_{7} \mathrm{H}_{14} \mathrm{O}$ & 114 & 0.30 & 0.46 \\
\hline 35 & 8.93 & m-xylene & $\mathrm{C}_{8} \mathrm{H}_{10}$ & 106 & - & 0.26 \\
\hline 36 & 9.14 & (Z)-4-Heptenal & $\mathrm{C}_{7} \mathrm{H}_{12} \mathrm{O}$ & 112 & 0.12 & - \\
\hline 37 & 9.17 & Heptanal & $\mathrm{C}_{7} \mathrm{H}_{14} \mathrm{O}$ & 114 & - & 2.74 \\
\hline 38 & 9.2 & 3-Methyl-2-Hexanol & $\mathrm{C}_{7} \mathrm{H}_{16} \mathrm{O}$ & 116 & 1.22 & - \\
\hline 39 & 9.94 & Methyl caproate & $\mathrm{C}_{7} \mathrm{H}_{14} \mathrm{O}_{2}$ & 130 & 0.08 & - \\
\hline 40 & 10 & 3-Methyl-4-Heptanone & $\mathrm{C}_{8} \mathrm{H}_{16} \mathrm{O}$ & 128 & 0.15 & - \\
\hline 41 & 11.28 & 3-methyl-4-Heptanol & $\mathrm{C}_{8} \mathrm{H}_{18} \mathrm{O}$ & 130 & 0.28 & - \\
\hline 42 & 11.38 & 2-methyl-4-Heptanol & $\mathrm{C}_{8} \mathrm{H}_{18} \mathrm{O}$ & 130 & 0.27 & - \\
\hline 43 & 11.42 & Heptanol & $\mathrm{C}_{7} \mathrm{H}_{16} \mathrm{O}$ & 116 & - & 0.48 \\
\hline 44 & 11.71 & 1-Octen-3-ol & $\mathrm{C}_{8} \mathrm{H}_{16} \mathrm{O}$ & 128 & 0.10 & 0.44 \\
\hline 45 & 11.79 & 2,3-Octanedione & $\mathrm{C}_{8} \mathrm{H}_{14} \mathrm{O}_{2}$ & 142 & 0.16 & 0.36 \\
\hline 46 & 11.84 & 6-methyl-5-Hepten-2-one & $\mathrm{C}_{8} \mathrm{H}_{14} \mathrm{O}$ & 126 & - & 0.56 \\
\hline 47 & 12.04 & 2-Pentyl-Furan & $\mathrm{C}_{9} \mathrm{H}_{14} \mathrm{O}$ & 138 & 0.53 & 2.00 \\
\hline 48 & 12.2 & 3-Octanol & $\mathrm{C}_{8} \mathrm{H}_{18} \mathrm{O}$ & 130 & 0.45 & - \\
\hline 49 & 12.27 & Ethyl Caproate & $\mathrm{C}_{8} \mathrm{H}_{16} \mathrm{O}_{2}$ & 144 & - & 0.80 \\
\hline 50 & 12.38 & Octanal & $\mathrm{C}_{8} \mathrm{H}_{16} \mathrm{O}$ & 128 & 0.99 & 0.73 \\
\hline 51 & 13.02 & (S)-3-ethyl-4-Methylpentanol & $\mathrm{C}_{8} \mathrm{H}_{18} \mathrm{O}$ & 130 & 0.37 & - \\
\hline 52 & 13.24 & 2-ethyl-1-Hexanol & $\mathrm{C}_{8} \mathrm{H}_{18} \mathrm{O}$ & 130 & 1.95 & 0.16 \\
\hline 53 & 13.28 & 1,8-Cineole & $\mathrm{C}_{10} \mathrm{H}_{18} \mathrm{O}$ & 154 & 0.89 & - \\
\hline 54 & 13.56 & 3-Octen-2-one & $\mathrm{C}_{8} \mathrm{H}_{14} \mathrm{O}$ & 126 & - & 0.22 \\
\hline 55 & 13.7 & Nitrohexane & $\mathrm{C}_{6} \mathrm{H}_{13} \mathrm{NO}$ & 131 & - & 0.48 \\
\hline 56 & 14.17 & (E)-2-Octenal & ${ }^{2} \mathrm{C}_{8} \mathrm{H}_{14} \mathrm{O}$ & 126 & - & 0.26 \\
\hline 57 & 14.61 & (E,E)-3,5-Octadien-2-one & $\mathrm{C}_{8} \mathrm{H}_{12} \mathrm{O}$ & 124 & - & 0.72 \\
\hline 58 & 14.81 & Allyl disulfide & $\mathrm{C}_{6} \mathrm{H}_{10} \mathrm{~S}_{2}$ & 146 & 0.29 & 0.34 \\
\hline 59 & 15.17 & 2-Nonanone & $\mathrm{C}_{9} \mathrm{H}_{18} \mathrm{O}$ & 142 & - & 0.93 \\
\hline 60 & 15.46 & Linalool & $\mathrm{C}_{10} \mathrm{H}_{18} \mathrm{O}$ & 154 & 0.61 & - \\
\hline 61 & 15.54 & Nonanal & $\mathrm{C}_{9} \mathrm{H}_{18} \mathrm{O}$ & 142 & 2.69 & 17.80 \\
\hline 62 & 16.82 & Camphor & $\mathrm{C}_{10} \mathrm{H}_{16} \mathrm{O}$ & 152 & 0.64 & 0.26 \\
\hline 63 & 17.29 & (E)-2-Nonenal & $\mathrm{C}_{9} \mathrm{H}_{16} \mathrm{O}$ & 140 & - & 1.53 \\
\hline 64 & 17.51 & endo-Borneol & $\mathrm{C}_{10} \mathrm{H}_{18} \mathrm{O}$ & 154 & 0.44 & 1.14 \\
\hline 65 & 17.7 & Nonanol & $\mathrm{C}_{9} \mathrm{H}_{20} \mathrm{O}$ & 144 & - & 1.08 \\
\hline 66 & 18.41 & Dodecane & $\mathrm{C}_{12} \mathrm{H}_{26}$ & 156 & - & 0.14 \\
\hline
\end{tabular}




\begin{tabular}{|c|c|c|c|c|c|c|}
\hline 67 & 18.59 & Decanal & $\mathrm{C}_{10} \mathrm{H}_{20} \mathrm{O}$ & 156 & 0.27 & 2.46 \\
\hline 68 & 19.99 & 1-butoxy-1-(2-methylpropoxy)-Butane & $\mathrm{C}_{12} \mathrm{H}_{26} \mathrm{O}_{2}$ & 204 & 0.12 & - \\
\hline 69 & 20.9 & Bornyl acetate & $\mathrm{C}_{12} \mathrm{H}_{20} \mathrm{O}_{2}$ & 196 & 0.11 & - \\
\hline 70 & 21.11 & 2-Vndecanone & $\mathrm{C}_{11} \mathrm{H}_{20} \mathrm{O}$ & 170 & - & 0.04 \\
\hline 71 & 21.24 & Tridecane & $\mathrm{C}_{13} \mathrm{H}_{18}$ & 184 & - & 0.18 \\
\hline 72 & 22.58 & $\alpha$-Copaene & $\mathrm{C}_{15} \mathrm{H}_{24}$ & 204 & - & 0.21 \\
\hline 73 & 22.69 & $\alpha$-Cubebene & $\mathrm{C}_{15} \mathrm{H}_{24}$ & 204 & 1.36 & 0.55 \\
\hline 74 & 23.42 & $\alpha$-Ylangene & $\mathrm{C}_{15} \mathrm{H}_{24}$ & 204 & 0.42 & 0.19 \\
\hline 75 & 23.81 & $\beta$-Elemene & $\mathrm{C}_{15} \mathrm{H}_{24}$ & 204 & 0.38 & - \\
\hline 76 & 23.89 & Tetradecane & $\mathrm{C}_{14} \mathrm{H}_{30}$ & 198 & 0.22 & 0.45 \\
\hline 77 & 24.27 & Junipene & $\mathrm{C}_{15} \mathrm{H}_{24}$ & 204 & - & 0.23 \\
\hline 78 & 24.59 & (E)-Caryophyllene & $\mathrm{C}_{15} \mathrm{H}_{24}$ & 204 & 0.28 & 0.24 \\
\hline 79 & 24.92 & Calarene & $\mathrm{C}_{15} \mathrm{H}_{24}$ & 204 & 0.15 & - \\
\hline 80 & 25.14 & $\alpha$-Garjunene & $\mathrm{C}_{15} \mathrm{H}_{24}$ & 204 & 0.13 & - \\
\hline 81 & 25.36 & (E)- $\beta$-farnesene & $\mathrm{C}_{15} \mathrm{H}_{24}$ & 204 & 1.96 & 0.68 \\
\hline 82 & 25.97 & Selin-4,7(11)-diene & $\mathrm{C}_{15} \mathrm{H}_{24}$ & 204 & 1.14 & 0.31 \\
\hline 83 & 26.28 & Alloaromadendrene & $\mathrm{C}_{15} \mathrm{H}_{24}$ & 204 & 0.45 & - \\
\hline 84 & 26.5 & $\alpha$-Selinene & $\mathrm{C}_{15} \mathrm{H}_{24}$ & 204 & 0.98 & 0.34 \\
\hline 85 & 26.67 & Hexadecanol & $\mathrm{C}_{16} \mathrm{H}_{34} \mathrm{O}$ & 242 & 4.38 & 0.60 \\
\hline 86 & 27.12 & 1s,(Z)-Calamene & $\mathrm{C}_{15} \mathrm{H}_{22}$ & 202 & 2.72 & 0.66 \\
\hline 87 & 27.36 & Cadina-1,4-diene & $\mathrm{C}_{15} \mathrm{H}_{24}$ & 204 & 0.34 & - \\
\hline 88 & 27.64 & $\alpha$-Calacorene & $\mathrm{C}_{15} \mathrm{H}_{20}$ & 200 & 0.14 & - \\
\hline 89 & 28.77 & Hexadecane & $\mathrm{C}_{16} \mathrm{H}_{34}$ & 226 & 0.47 & 1.25 \\
\hline 90 & 29.08 & Tetradecanal & $\mathrm{C}_{14} \mathrm{H}_{28} \mathrm{O}$ & 212 & 9.08 & 2.30 \\
\hline 91 & 31.02 & Heptadecane & $\mathrm{C}_{17} \mathrm{H}_{36}$ & 240 & 0.25 & 0.76 \\
\hline 92 & 31.14 & 2,6-dimethyl-Heptadecane & $\mathrm{C}_{19} \mathrm{H}_{40}$ & 268 & 0.24 & 0.41 \\
\hline 93 & 31.39 & Hexadecanal & $\mathrm{C}_{16} \mathrm{H}_{32} \mathrm{O}$ & 240 & 32.3 & 8.51 \\
\hline 94 & 33.17 & Octadecane & $\mathrm{C}_{18} \mathrm{H}_{38}$ & 254 & 0.14 & - \\
\hline 95 & 35.11 & 9,12-Octadecadienal & $\mathrm{C}_{18} \mathrm{H}_{32 \mathrm{O}}$ & 264 & 0.51 & - \\
\hline 96 & 35.22 & Nonadecane & $\mathrm{C}_{18} \mathrm{H}_{38}$ & 254 & 1.16 & - \\
\hline 97 & 35.79 & Methyl plamitate & $\mathrm{C}_{17} \mathrm{H}_{34} \mathrm{O}_{2}$ & 270 & 0.20 & - \\
\hline
\end{tabular}

Table 2 Volatiles from fresh and dry pseudostem of Musa basjoo

\begin{tabular}{|c|c|c|c|c|c|c|}
\hline \multirow[t]{2}{*}{ No. } & \multirow{2}{*}{$\begin{array}{l}\text { Retention } \\
\text { time(min) }\end{array}$} & \multirow[t]{2}{*}{ Compound name } & \multirow[t]{2}{*}{ Formula } & \multirow{2}{*}{$\begin{array}{l}\text { Molecular } \\
\text { Weight }\end{array}$} & \multicolumn{2}{|c|}{ The percentage (\%) } \\
\hline & & & & & Fresh samples & $\begin{array}{l}\text { Dry } \\
\text { samples }\end{array}$ \\
\hline 1 & 2.02 & Ethanal & $\mathrm{C}_{2} \mathrm{H}_{4} \mathrm{O}$ & 44 & - & 2.69 \\
\hline 2 & 2.14 & Ethanol & $\mathrm{C}_{2} \mathrm{H}_{6} \mathrm{O}$ & 46 & 2.3 & 10.23 \\
\hline 3 & 2.4 & Methyl acetate & $\mathrm{C}_{3} \mathrm{H}_{6} \mathrm{O}$ & 74 & 1.65 & 0.24 \\
\hline 4 & 2.55 & Isobutanal & $\mathrm{C}_{4} \mathrm{H}_{8} \mathrm{O}$ & 72 & - & 0.41 \\
\hline 5 & 2.63 & (E)-2-Butanal & $\mathrm{C}_{4} \mathrm{H}_{6} \mathrm{O}$ & 70 & - & 0.07 \\
\hline 6 & 2.82 & 2-Butanone & $\mathrm{C}_{4} \mathrm{H}_{8} \mathrm{O}$ & 72 & - & 0.10 \\
\hline 7 & 2.98 & Ethyl acetate & $\mathrm{C}_{4} \mathrm{H}_{8} \mathrm{O}_{2}$ & 88 & 3.64 & 0.57 \\
\hline 8 & 3.12 & Isobutanol & $\mathrm{C}_{4} \mathrm{H}_{10} \mathrm{O}$ & 74 & - & 0.27 \\
\hline 9 & 3.39 & (Z)-2-Butenal & $\mathrm{C}_{4} \mathrm{H}_{6} \mathrm{O}$ & 70 & - & 0.04 \\
\hline 10 & 3.42 & 3-methyl-Butenal & $\mathrm{C}_{5} \mathrm{H}_{10} \mathrm{O}$ & 86 & - & 0.77 \\
\hline 11 & 3.55 & 2-methyl-Butenal & $\mathrm{C}_{5} \mathrm{H}_{10} \mathrm{O}$ & 86 & - & 0.89 \\
\hline 12 & 3.57 & 1-Butanol & $\mathrm{C}_{4} \mathrm{H}_{10} \mathrm{O}$ & 74 & 0.75 & - \\
\hline 13 & 3.83 & 1-Penten-3-ol & $\mathrm{C}_{5} \mathrm{H}_{10} \mathrm{O}$ & 86 & - & 0.11 \\
\hline 14 & 3.88 & Triethylamine & $\mathrm{C}_{6} \mathrm{H}_{15} \mathrm{~N}$ & 101 & - & 0.19 \\
\hline 15 & 4.04 & Pentanal & $\mathrm{C}_{5} \mathrm{H}_{10} \mathrm{O}$ & 86 & 0.36 & 0.42 \\
\hline 16 & 4.31 & Ethyl propionate & $\mathrm{C}_{5} \mathrm{H}_{10} \mathrm{O}_{2}$ & 102 & - & 0.05 \\
\hline 17 & 4.61 & 2,4,5-trimethyl-1,3-dioxolane & $\mathrm{C}_{6} \mathrm{H}_{12} \mathrm{O}_{2}$ & 116 & - & 0.09 \\
\hline 18 & 4.68 & 1,1-diethoxy-Ethane & $\mathrm{C}_{6} \mathrm{H}_{14} \mathrm{O}_{2}$ & 118 & - & 0.07 \\
\hline
\end{tabular}




\begin{tabular}{|c|c|c|c|c|c|c|}
\hline 19 & 4.77 & 3-methyl-1-Butanol & $\mathrm{C}_{5} \mathrm{H}_{12} \mathrm{O}$ & 88 & - & 0.59 \\
\hline 20 & 4.85 & 2-methyl-1-Butanol & $\mathrm{C}_{5} \mathrm{H}_{12} \mathrm{O}$ & 88 & - & 0.49 \\
\hline 21 & 5.29 & Ethyl isobutyrate & $\mathrm{C}_{6} \mathrm{H}_{12} \mathrm{O}_{2}$ & 116 & - & 0.20 \\
\hline 22 & 5.52 & 1-Pentanol & $\mathrm{C}_{5} \mathrm{H}_{12} \mathrm{O}$ & 88 & 0.37 & 0.60 \\
\hline 23 & 5.92 & Diethyl carbonate & $\mathrm{C}_{5} \mathrm{H}_{10} \mathrm{O}_{3}$ & 118 & 0.32 & - \\
\hline 24 & 6.24 & Hexanal & $\mathrm{C}_{6} \mathrm{H}_{12} \mathrm{O}$ & 100 & 8.62 & 14.32 \\
\hline 25 & 6.66 & Butyl acetate & $\mathrm{C}_{6} \mathrm{H}_{12} \mathrm{O}_{2}$ & 116 & - & 0.16 \\
\hline 26 & 7.24 & 3-methyl-Hexan-2-one & $\mathrm{C}_{6} \mathrm{H}_{10} \mathrm{O}_{2}$ & 114 & - & 0.16 \\
\hline 27 & 7.41 & 3-Methyl-3-Hexanol & $\mathrm{C}_{7} \mathrm{H}_{16} \mathrm{O}$ & 116 & 0.13 & - \\
\hline 28 & 7.67 & Ethyl 2-methylbutyrate & $\mathrm{C}_{7} \mathrm{H}_{14} \mathrm{O}_{2}$ & 130 & - & 0.20 \\
\hline 29 & 7.78 & (Z)-3-Hexenal & $\mathrm{C}_{6} \mathrm{H}_{10} \mathrm{O}$ & 98 & 0.14 & 0.28 \\
\hline 30 & 8 & Ethylbenzene & $\mathrm{C}_{8} \mathrm{H}_{10}$ & 106 & - & 0.19 \\
\hline 31 & 8.26 & 1-Hexanol & $\mathrm{C}_{6} \mathrm{H}_{14} \mathrm{O}$ & 102 & 7.02 & 4.61 \\
\hline 32 & 8.45 & Isoamyl acetate & $\mathrm{C}_{7} \mathrm{H}_{14} \mathrm{O}_{2}$ & 130 & - & 0.15 \\
\hline 33 & 8.74 & $\begin{array}{l}\text { 5-methyl-3-methylene-5- } \\
\text { Hexen-2-one }\end{array}$ & $\mathrm{C}_{8} \mathrm{H}_{12} \mathrm{O}$ & 124 & - & 0.08 \\
\hline 34 & 8.86 & 2-Heptanone & $\mathrm{C}_{7} \mathrm{H}_{14} \mathrm{O}$ & 114 & 1.65 & 0.26 \\
\hline 35 & 8.89 & Styrene & $\mathrm{C}_{8} \mathrm{H}_{8}$ & 104 & - & 0.52 \\
\hline 36 & 8.93 & m-xylene & $\mathrm{C}_{8} \mathrm{H}_{10}$ & 106 & - & 0.24 \\
\hline 37 & 9.14 & (Z)-4-Heptenal & $\mathrm{C}_{7} \mathrm{H}_{12} \mathrm{O}$ & 112 & 0.11 & - \\
\hline 38 & 9.19 & 3-Methyl-2-Hexanol & $\mathrm{C}_{7} \mathrm{H}_{16} \mathrm{O}$ & 116 & 3.56 & - \\
\hline 39 & 9.19 & Heptanal & $\mathrm{C}_{7} \mathrm{H}_{14} \mathrm{O}$ & 114 & - & 6.98 \\
\hline 40 & 9.61 & Methoxy-phenyl-oxime & $\mathrm{C}_{8} \mathrm{H}_{9} \mathrm{NO}_{2}$ & 151 & - & 0.14 \\
\hline 41 & 9.99 & 3-Methyl-4-Heptanone & $\mathrm{C}_{8} \mathrm{H}_{16} \mathrm{O}$ & 128 & 0.18 & - \\
\hline 42 & 10.93 & (E)-2-Heptenal & $\mathrm{C}_{7} \mathrm{H}_{12} \mathrm{O}$ & 112 & - & 0.38 \\
\hline 43 & 11.41 & Heptanol & $\mathrm{C}_{7} \mathrm{H}_{16} \mathrm{O}$ & 116 & - & 0.59 \\
\hline 44 & 11.7 & 1-Octen-3-ol & $\mathrm{C}_{8} \mathrm{H}_{16} \mathrm{O}$ & 128 & - & 0.73 \\
\hline 45 & 11.79 & 2,3-Octanedione & $\mathrm{C}_{8} \mathrm{H}_{14} \mathrm{O}_{2}$ & 142 & 0.46 & 0.43 \\
\hline 46 & 11.88 & 6-methyl-5-Hepten-2-one & $\mathrm{C}_{8} \mathrm{H}_{14} \mathrm{O}$ & 126 & - & 0.54 \\
\hline 47 & 12.05 & 2-Pentyl-Furan & $\mathrm{C}_{9} \mathrm{H}_{14} \mathrm{O}$ & 138 & 1.59 & 0.90 \\
\hline 48 & 12.28 & Ethyl Caproate & $\mathrm{C}_{8} \mathrm{H}_{16} \mathrm{O}_{2}$ & 144 & - & 1.15 \\
\hline 49 & 12.38 & Octanal & $\mathrm{C}_{8} \mathrm{H}_{16} \mathrm{O}$ & 128 & 3.11 & 1.98 \\
\hline 50 & 12.65 & (E)- $\beta$-Ocimene & $\mathrm{C}_{10} \mathrm{H}_{16}$ & 136 & 0.25 & - \\
\hline 51 & 13.25 & 2-ethyl-1-Hexanol & $\mathrm{C}_{8} \mathrm{H}_{18} \mathrm{O}$ & 130 & 4.89 & 0.22 \\
\hline 52 & 13.29 & 1,8-Cineole & $\mathrm{C}_{10} \mathrm{H}_{18} \mathrm{O}$ & 154 & 1.73 & - \\
\hline 53 & 13.53 & 3-Ochen-2-one & $\mathrm{C}_{8} \mathrm{H}_{14} \mathrm{O}$ & 126 & - & 0.93 \\
\hline 54 & 14.13 & (E)-2-Octenal & $\mathrm{C}_{8} \mathrm{H}_{14} \mathrm{O}$ & 126 & - & 0.51 \\
\hline 55 & 14.46 & (E)-Sabinene hydrate & $\mathrm{C}_{10} \mathrm{H}_{18} \mathrm{O}$ & 154 & 0.22 & - \\
\hline 56 & 14.54 & $(\mathrm{E}, \mathrm{E})-3,5$-Octadien-2-one & $\mathrm{C}_{8} \mathrm{H}_{12} \mathrm{O}$ & 124 & - & 1.03 \\
\hline 57 & 14.81 & Allyl disulfide & $\mathrm{C}_{6} \mathrm{H}_{10} \mathrm{~S}_{2}$ & 146 & - & 0.28 \\
\hline 58 & 15.17 & 2-Nonanone & $\mathrm{C}_{9} \mathrm{H}_{18} \mathrm{O}$ & 142 & - & 0.58 \\
\hline 59 & 15.27 & $\begin{array}{l}\text { 2-Methoxy-3-isopropyl } \\
\text { pyrazine }\end{array}$ & $\mathrm{C}_{8} \mathrm{H}_{12} \mathrm{~N}_{2} \mathrm{O}$ & 152 & 0.09 & - \\
\hline 60 & 15.47 & Linalool & $\mathrm{C}_{10} \mathrm{H}_{18} \mathrm{O}$ & 154 & 12.12 & - \\
\hline 61 & 15.55 & Nonanal & $\mathrm{C}_{9} \mathrm{H}_{18} \mathrm{O}$ & 142 & 6.7 & 30.88 \\
\hline 62 & 16.49 & (Z)-Limonene oxide & $\mathrm{C}_{10} \mathrm{H}_{16} \mathrm{O}$ & 152 & 0.28 & - \\
\hline 63 & 16.83 & Camphor & $\mathrm{C}_{10} \mathrm{H}_{16} \mathrm{O}$ & 152 & 2.07 & 0.13 \\
\hline 64 & 17.24 & (E)-2-Nonenal & $\mathrm{C}_{9} \mathrm{H}_{16} \mathrm{O}$ & 140 & - & 0.62 \\
\hline 65 & 17.52 & endo-Borneol & $\mathrm{C}_{10} \mathrm{H}_{18} \mathrm{O}$ & 154 & 1.45 & 0.88 \\
\hline 66 & 17.66 & Nonanol & $\mathrm{C}_{9} \mathrm{H}_{20} \mathrm{O}$ & 144 & - & 0.33 \\
\hline 67 & 18.27 & $\alpha$-Terpineol & $\mathrm{C}_{10} \mathrm{H}_{18} \mathrm{O}$ & 154 & 0.27 & - \\
\hline 68 & 18.41 & Dodecane & $\mathrm{C}_{12} \mathrm{H}_{26}$ & 170 & 0.22 & 0.11 \\
\hline 69 & 18.6 & Decanal & $\mathrm{C}_{10} \mathrm{H}_{20} \mathrm{O}$ & 156 & 1.08 & 4.85 \\
\hline 70 & 19.08 & $\begin{array}{l}\text { 1,3,4-trimethyl-3-cyclohexene- } \\
\text { 1-carboxaldehyde }\end{array}$ & $\mathrm{C}_{10} \mathrm{H}_{16} \mathrm{O}$ & 152 & - & 0.14 \\
\hline 71 & 20.91 & Bornyl acetate & $\mathrm{C}_{12} \mathrm{H}_{20} \mathrm{O}_{2}$ & 196 & 0.12 & - \\
\hline 72 & 21.09 & 2-Vndecanone & $\mathrm{C}_{11} \mathrm{H}_{20} \mathrm{O}$ & 170 & - & 0.11 \\
\hline 73 & 21.24 & Tridecane & $\mathrm{C}_{13} \mathrm{H}_{28}$ & 184 & 0.10 & 0.07 \\
\hline
\end{tabular}




\begin{tabular}{lllllll}
\hline 74 & 21.45 & Dodecanal & $\mathrm{C}_{12} \mathrm{H}_{24} \mathrm{O}$ & 184 & - & 1.27 \\
75 & 22.59 & $\alpha$-Copaene & $\mathrm{C}_{15} \mathrm{H}_{24}$ & 204 & 0.12 & 0.04 \\
76 & 22.7 & $\alpha$-Cubebene & $\mathrm{C}_{15} \mathrm{H}_{24}$ & 204 & 0.27 & 0.06 \\
77 & 23.83 & $\beta$-Elemene & $\mathrm{C}_{15} \mathrm{H}_{24}$ & 204 & 0.40 & - \\
78 & 23.9 & Tetradecane & $\mathrm{C}_{14} \mathrm{H}_{30}$ & 198 & 0.40 & 0.09 \\
79 & 24.26 & Junipene & $\mathrm{C}_{15} \mathrm{H}_{24}$ & 204 & - & 0.12 \\
80 & 24.69 & $\alpha$-Ionone & $\mathrm{C}_{13} \mathrm{H}_{20} \mathrm{O}$ & 192 & - & 0.29 \\
81 & 26.18 & $\beta$-Ionone & $\mathrm{C}_{13} \mathrm{H}_{20} \mathrm{O}$ & 192 & - & 0.19 \\
82 & 26.7 & Seychellene & $\mathrm{C}_{15} \mathrm{H}_{24}$ & 204 & 0.44 & 0.16 \\
83 & 27.13 & Calamenene & $\mathrm{C}_{15} \mathrm{H}_{22}$ & 202 & 0.22 & - \\
84 & 28.78 & Hexadecane & $\mathrm{C}_{16} \mathrm{H}_{34}$ & 226 & 1.02 & 0.21 \\
85 & 29.11 & Tetradecanal & $\mathrm{C}_{14} \mathrm{H}_{28} \mathrm{O}$ & 212 & 1.61 & - \\
86 & 31.03 & Heptadecane & $\mathrm{C}_{17} \mathrm{H}_{36}$ & 240 & 1.55 & 0.09 \\
87 & 31.16 & 2,6-dimethyl-Heptadecane & $\mathrm{C}_{19} \mathrm{H}_{40}$ & 268 & 0.53 & 0.06 \\
88 & 31.39 & Hexadecanal & $\mathrm{C}_{16} \mathrm{H}_{32} \mathrm{O}$ & 240 & 10.98 & - \\
89 & 31.54 & Methyl myristate & $\mathrm{C}_{15} \mathrm{H}_{30} \mathrm{O}_{2}$ & 242 & 0.70 & - \\
90 & 33.17 & Octadecane & $\mathrm{C}_{18} \mathrm{H}_{38}$ & 254 & 0.26 & - \\
91 & 35.23 & Nonadecane & $\mathrm{C}_{19} \mathrm{H}_{40}$ & 268 & 0.30 & - \\
92 & 35.78 & Methyl palmitate & $\mathrm{C}_{17} \mathrm{H}_{34} \mathrm{O}_{2}$ & 270 & 1.72 & - \\
93 & 37.1 & $\mathrm{C}_{17} \mathrm{H}_{34} \mathrm{O}_{2}$ & 270 & 0.12 & - \\
94 & 38.57 & Ethyl pentadecanoate & $\mathrm{C}_{21} \mathrm{H}_{42}$ & 294 & 0.60 & - \\
95 & 39.05 & 10-Heneicosene & $\mathrm{C}_{21} \mathrm{H}_{44}$ & 296 & 0.57 & - \\
96 & 42.11 & Heneicosane & $\mathrm{C}_{23} \mathrm{H}_{46}$ & 322 & 0.53 & - \\
\hline
\end{tabular}

Table 3 Volatiles from fresh and dry leaf of Musa basjoo

\begin{tabular}{|c|c|c|c|c|c|c|}
\hline \multirow[t]{2}{*}{ No. } & \multirow{2}{*}{$\begin{array}{l}\text { Retention } \\
\text { time(min) }\end{array}$} & \multirow[t]{2}{*}{ Compound name } & \multirow[t]{2}{*}{ Formula } & \multirow{2}{*}{$\begin{array}{l}\text { Molecular } \\
\text { Weight }\end{array}$} & \multicolumn{2}{|c|}{ The percentage $(\%)$} \\
\hline & & & & & Fresh samples & Dry samples \\
\hline 1 & 1.98 & Ethanal & $\mathrm{C}_{2} \mathrm{H}_{4} \mathrm{O}$ & 44 & - & 8.95 \\
\hline 2 & 2.1 & Ethanol & $\mathrm{C}_{2} \mathrm{H}_{6} \mathrm{O}$ & 46 & - & 11.91 \\
\hline 3 & 2.39 & Methy acetate & $\mathrm{C}_{3} \mathrm{H}_{6} \mathrm{O}_{2}$ & 74 & 1.68 & - \\
\hline 4 & 2.53 & Isobutanal & $\mathrm{C}_{4} \mathrm{H}_{8} \mathrm{O}$ & 72 & 1.23 & 6.96 \\
\hline 5 & 2.79 & 2-Butanone & $\mathrm{C}_{4} \mathrm{H}_{8} \mathrm{O}$ & 72 & - & 0.60 \\
\hline 6 & 2.99 & Ethyl acetate & $\mathrm{C}_{4} \mathrm{H}_{8} \mathrm{O}_{2}$ & 88 & - & 0.41 \\
\hline 7 & 3.11 & Isobutanol & $\mathrm{C}_{4} \mathrm{H}_{10} \mathrm{O}$ & 74 & - & 0.76 \\
\hline 8 & 3.39 & (Z)-2-Butenal & $\mathrm{C}_{4} \mathrm{H}_{6} \mathrm{O}$ & 70 & - & 6.68 \\
\hline 9 & 3.41 & 3-methyl-Butanal & $\mathrm{C}_{5} \mathrm{H}_{6} \mathrm{O}$ & 86 & 9.35 & 1.81 \\
\hline 10 & 3.54 & 2-methyl-Butanal & $\mathrm{C}_{5} \mathrm{H}_{6} \mathrm{O}$ & 86 & 4.30 & 10.12 \\
\hline 11 & 3.81 & 1-Penten-3-ol & $\mathrm{C}_{5} \mathrm{H}_{10} \mathrm{O}$ & 86 & - & 0.73 \\
\hline 12 & 3.87 & Triethyl amine & $\mathrm{C}_{6} \mathrm{H}_{15} \mathrm{~N}$ & 101 & - & 0.44 \\
\hline 13 & 4.03 & Pentanol & $\mathrm{C}_{5} \mathrm{H}_{10} \mathrm{O}$ & 86 & - & 0.77 \\
\hline 14 & 4.05 & Pentanal & $\mathrm{C}_{5} \mathrm{H}_{10} \mathrm{O}$ & 86 & 4.14 & - \\
\hline 15 & 4.09 & 2-ethyl-furan & $\mathrm{C}_{6} \mathrm{H}_{8} \mathrm{O}$ & 96 & - & 1.00 \\
\hline 16 & 4.6 & 2,4,5-trimethyl-1,3-dioxolane & $\mathrm{C}_{6} \mathrm{H}_{12} \mathrm{O}_{2}$ & 116 & - & 0.10 \\
\hline 17 & 4.67 & 1,1-diethoxy-Ethane & $\mathrm{C}_{6} \mathrm{H}_{14} \mathrm{O}_{2}$ & 118 & - & 0.12 \\
\hline 18 & 4.77 & 3-methyl-1-Butanol & $\mathrm{C}_{5} \mathrm{H}_{12} \mathrm{O}$ & 88 & 1.66 & 1.37 \\
\hline 19 & 4.85 & 2-methyl-1-Butanol & $\mathrm{C}_{5} \mathrm{H}_{12} \mathrm{O}$ & 88 & 1.02 & 1.92 \\
\hline 20 & 5.27 & Ethyl isobutyrate & $\mathrm{C}_{6} \mathrm{H}_{12} \mathrm{O}_{2}$ & 116 & - & 0.64 \\
\hline 21 & 5.53 & 1-Pentanol & $\mathrm{C}_{5} \mathrm{H}_{12} \mathrm{O}$ & 88 & - & 0.19 \\
\hline 22 & 6.23 & Hexanal & $\mathrm{C}_{6} \mathrm{H}_{12} \mathrm{O}$ & 100 & 5.67 & 10.48 \\
\hline 23 & 7.23 & 3-methyl-Hexan-2-one & $\mathrm{C}_{6} \mathrm{H}_{10} \mathrm{O}_{2}$ & 114 & - & 0.79 \\
\hline 24 & 7.67 & Ethyl 2-methylbutyrate & $\mathrm{C}_{7} \mathrm{H}_{14} \mathrm{O}_{2}$ & 130 & - & 0.22 \\
\hline 25 & 7.75 & (Z)-3-Hexenal & $\mathrm{C}_{6} \mathrm{H}_{10} \mathrm{O}$ & 98 & 17.33 & 3.88 \\
\hline 26 & 7.99 & Ethyl benzene & $\mathrm{C}_{8} \mathrm{H}_{10}$ & 106 & - & 1.23 \\
\hline 27 & 8.29 & 1-Hexanol & $\mathrm{C}_{6} \mathrm{H}_{14} \mathrm{O}$ & 102 & - & 2.12 \\
\hline 28 & 8.9 & Styrene & $\mathrm{C}_{8} \mathrm{H}_{8}$ & 104 & - & 0.43 \\
\hline 29 & 8.93 & m-xylene & $\mathrm{C}_{8} \mathrm{H}_{10}$ & 106 & - & 0.46 \\
\hline 30 & 9.18 & Heptanal & $\mathrm{C}_{7} \mathrm{H}_{14} \mathrm{O}$ & 114 & - & 4.07 \\
\hline 31 & 9.2 & 3-Methy-2-Hexanol & $\mathrm{C}_{7} \mathrm{H}_{16} \mathrm{O}$ & 116 & 3.30 & - \\
\hline
\end{tabular}




\begin{tabular}{|c|c|c|c|c|c|c|}
\hline 32 & 10.99 & (E)-2-Heptenal & $\mathrm{C}_{7} \mathrm{H}_{12} \mathrm{O}$ & 112 & - & 0.47 \\
\hline 33 & 11.72 & 1-Octen-3-ol & $\mathrm{C}_{8} \mathrm{H}_{16} \mathrm{O}$ & 128 & - & 0.58 \\
\hline 34 & 11.79 & 2,3-Octanedione & $\mathrm{C}_{8} \mathrm{H}_{14} \mathrm{O}_{2}$ & 142 & - & 0.35 \\
\hline 35 & 11.9 & 6-methyl-5-Hepten-2-one & $\mathrm{C}_{8} \mathrm{H}_{14} \mathrm{O}$ & 126 & - & 0.71 \\
\hline 36 & 12.06 & 2-Pentyl-Furan & $\mathrm{C}_{9} \mathrm{H}_{14} \mathrm{O}$ & 138 & 1.13 & 1.10 \\
\hline 37 & 12.28 & Ethyl Caproate & $\mathrm{C}_{8} \mathrm{H}_{16} \mathrm{O}_{2}$ & 144 & - & 0.80 \\
\hline 38 & 12.4 & Octanal & $\mathrm{C}_{8} \mathrm{H}_{16} \mathrm{O}$ & 128 & - & 0.70 \\
\hline 39 & 14.01 & $\begin{array}{l}\text { 2,4,4-trimethyl-2-Cyclohexen-1- } \\
\text { ol }\end{array}$ & $\mathrm{C}_{9} \mathrm{H}_{16} \mathrm{O}$ & 140 & - & 0.34 \\
\hline 40 & 14.83 & Allyl disulfide & $\mathrm{C}_{6} \mathrm{H}_{10} \mathrm{~S}_{2}$ & 146 & - & 0.32 \\
\hline 41 & 15.47 & Linalool & $\mathrm{C}_{10} \mathrm{H}_{18} \mathrm{O}$ & 154 & 0.29 & - \\
\hline 42 & 15.64 & Nonanal & $\mathrm{C}_{9} \mathrm{H}_{18} \mathrm{O}$ & 142 & 1.42 & 3.34 \\
\hline 43 & 15.73 & Neoisomenthol & $\mathrm{C}_{10} \mathrm{H}_{20} \mathrm{O}$ & 156 & - & 3.94 \\
\hline 44 & 16.82 & Camphor & $\mathrm{C}_{10} \mathrm{H}_{16} \mathrm{O}$ & 152 & 0.87 & - \\
\hline 45 & 17.52 & Endo-Borneol & $\mathrm{C}_{10} \mathrm{H}_{18} \mathrm{O}$ & 154 & - & 0.23 \\
\hline 46 & 19.08 & $\begin{array}{l}\text { 1,3,4-trimethyl-3-cyclohexene-1- } \\
\text { carboxaldehyde }\end{array}$ & $\mathrm{C}_{10} \mathrm{H}_{16} \mathrm{O}$ & 152 & 1.45 & 0.68 \\
\hline 47 & 22.58 & $\alpha$-Copaene & $\mathrm{C}_{15} \mathrm{H}_{24}$ & 204 & - & 0.06 \\
\hline 48 & 22.69 & $\alpha$-Cubebene & $\mathrm{C}_{15} \mathrm{H}_{24}$ & 204 & 0.41 & - \\
\hline 49 & 23.19 & (+)-Cycloisosativene & $\mathrm{C}_{15} \mathrm{H}_{24}$ & 204 & 0.54 & - \\
\hline 50 & 23.42 & $\alpha$-Ylangene & $\mathrm{C}_{15} \mathrm{H}_{24}$ & 204 & 0.97 & - \\
\hline 51 & 23.82 & $\beta$-Elemene & $\mathrm{C}_{15} \mathrm{H}_{24}$ & 204 & 0.61 & - \\
\hline 52 & 24.26 & Junipene & $\mathrm{C}_{15} \mathrm{H}_{24}$ & 204 & - & 0.05 \\
\hline 53 & 24.69 & $\alpha$-Ionone & $\mathrm{C}_{13} \mathrm{H}_{20} \mathrm{O}$ & 192 & 2.41 & 2.30 \\
\hline 54 & 26.18 & $\beta$-Ionone & $\mathrm{C}_{13} \mathrm{H}_{20} \mathrm{O}$ & 192 & 3.03 & 1.20 \\
\hline 55 & 26.57 & $\alpha$-Muurolene & $\mathrm{C}_{15} \mathrm{H}_{24}$ & 204 & 1.30 & - \\
\hline 56 & 27.12 & $\sigma$-Cadinene & $\mathrm{C}_{15} \mathrm{H}_{24}$ & 204 & 1.41 & - \\
\hline 57 & 28.8 & Hexadecane & $\mathrm{C} 16 \mathrm{H}_{34}$ & 226 & 1.00 & - \\
\hline 58 & 31.05 & Heptadecane & $\mathrm{C}_{17} \mathrm{H}_{36}$ & 240 & 0.58 & - \\
\hline 59 & 31.17 & 2,6-dimethyl-Heptadecane & $\mathrm{C}_{19} \mathrm{H}_{40}$ & 268 & 0.62 & - \\
\hline 60 & 31.44 & Hexadecanal & $\mathrm{C}_{16} \mathrm{H}_{32} \mathrm{O}$ & 240 & 17.85 & - \\
\hline 61 & 33.18 & Octadecane & $\mathrm{C}_{18} \mathrm{H}_{38}$ & 254 & 0.30 & - \\
\hline 62 & 35.81 & Nonadecane & $\mathrm{C}_{19} \mathrm{H}_{40}$ & 268 & 7.17 & - \\
\hline 63 & 39.06 & Heneicosane & $\mathrm{C}_{21} \mathrm{H}_{44}$ & 296 & 0.37 & - \\
\hline 64 & 39.6 & Methyl Stearate & $\mathrm{C}_{19} \mathrm{H}_{38} \mathrm{O}$ & 298 & 0.42 & - \\
\hline
\end{tabular}

The indentified volatile compounds of dry Musa basjoo pseudostem were more than the fresh pseudostem samples, 73 compounds were indentified, accounting for the $98.33 \%$ of the total volatiles, The main constituents were Nonanal (30.88\%), Heptanal (6.98\%), Hexanal (14.32\%), Ethanol (10.23\%), 1-Hexanol (4.61\%), Ethanal (2.69\%). In the fresh pseudostem, only 52 compounds were indentified, accounting for $89.89 \%$. Linalool (12.12\%), Hexadecanal (10.98\%), Hexanal (8.62\%), 1-Hexanol $(7.02 \%)$, Nonanal $(6.70 \%)$, and 2-ethyl-1-Hexanol $(4.89 \%)$ were the main constituents in the total volatiles. There were 26 mutual compounds in the fresh and dry Musa basjoo pseudostem. Alcohols and aldehydes were the main compositions in the Musa basjoo pseudostem volatiles. Esters in fresh Musa basjoo pseudostem were much higher than in the dry sample (table 2).

31 and 45 volatile compounds were detected in the fresh and dry Musa basjoo leaf, respectively. The content of identify compounds was higher than $93 \%$ in the total volatiles of both fresh and dry Musa basjoo leaf. The main constituents were Hexadecanal (17.85\%), (Z)-3-Hexenal (17.33\%), 3methyl-Butanal (9.35\%), Hexanal (5.67\%), 2-methyl-Butanal (4.30\%), and Pentanal (4.14\%). In the dry leaf, the main constituents included Ethanol (11.91\%), Hexanal (10.48\%), 2-methyl-Butanal $(10.12 \%)$, Ethanal (8.95\%), Isobutanal (6.96\%), (Z)-2-Butenal (6.68\%), and Heptanal (4.07\%). Neoisomenthol (3.94\%) (Z)-3-Hexenal (3.88\%), and Nonanal (3.34\%). The aldehydes and alcohols were the higher compounds in the fresh and dry leaf. The esters in fresh leaf were $9.27 \%$, while only $2.07 \%$ in the dry sample. It may be associated with the volatility of esters during the drying process (table 3 ). 
Comparison of the volatiles of fresh rhizome, pseudostem and leaf of Musa basjoo, only 14 compounds were mutual in the volatiles of six Musa basjoo parts. Comparison among fresh rhizome, pseudostem and leaf, the mutual compounds were 30, 18, 14 compounds, respectively. The main compounds were Hexadecanal and Hexhanal. 35 mutual compounds were detected in the dry rhizome, pseudostem and leaf samples. 59, 36, 40 compounds were identified as the mutual constituents in the volatiles of dry rhizome, pseudostem and leaf, respectively. Some main compounds, such as Ethanal, Hexanal, Nonanal, Ethanol, and 1-Hexanol were mutual in the dry rhizome, pseudostem and leaf, accounting for $50.70 \%, 62.73 \%$ and $36.8 \%$ of the identified compound in the three parts volatiles of Musa basjoo, respectively.

From the above, the results showed that there is a great difference between the fresh and dry (rhizome, pseudostem and leaf) samples. The volatile oil components among different parts of the Musa basjoo had significant differences, too. In the development and utilization of Musa basjoo volatile oils, the difference among the three parts volatiles is important. Volatile oils have antibacterial (Abdul et al. 2014), anti-inflammatory (Soares et al. 2014) and anti-tumor activity (Jiang et al. 2012) etc. Musa basjoo have a variety of pharmacological activities with rich resources, so more works should do on its pharmacological activities.

\section{Acknowledgements}

This work was supported by the project "Science and Technology Foundation of Guizhou Province (Qian-ke-he [2014] No. 2041)" and "Guiyang Medical College, Dr. Fund (2014)".

\section{References}

[1] Bao, J.\& Ran, M.X. 1999. The study, development and re-evaluation of the Miao people's medicines of Guizhou Province. Guiyang: Guizhou Science and Technology Press.

[2] Drug Administration of Guizhou Province. 2003. Herbs, national medicinal quality standards in Guizhou Province. Guiyang: Guizhou science and Technology Press.

[3] Editorial board of state administration of traditional Chinese medicine for Chinese herbal medicine. 1999. Chinese herbal medicine. Shanghai: Shanghai Science and Technology Press.

[4] Jiang, J. L.\& Ding, H. T.\& Su, X.\& Yuan, Y.J. 2012. Identification of anti-tumor ingredients in curcuma volatile oil based on composition-activity relationship. Chin. J. Anal. Chem. 40: 14881493.

[5] Laghari, A. H.\& Memon, S.\& Nelofar, A.\& Khan, K.M. 2014. Determination of volatile constituents and antimicrobial activity of camel thorn (Alhagi camelorum) flowers. Anal. Lett. 47(3): 413-421.

[6] Qian, H.B.\& Hao, J.J.\& Wang. X.P. 2012. Effect of effective parts from Musa basjoo on blood glucose and glucose tolerance in mice. Chin. J. Exp. Tradit. Med. Form. 18: 187-189.

[7] Qian, H.B.\& Sun, Y. \& Huang, J.\& Wang, X.P. 2010. Experimental study on antiinflammatory and analgesic effects of different extracts from Musa basjoo. Lishizhen Med. Mater. Med. Res. 21: 780-781.

[8] Soares, D. J.\& Walker\& Pignitter, M.\& Walker, J. M.\& Imboeck, J. M.\& Ehrnhoefer-Ressler, M. M.\& Somoza, V. 2014. Pitanga (Eugenia uniflora L.) fruit juice and two major constituents thereof exhibit anti-inflammatory properties in human gingival and oral gum epithelial cells. Food Funct. 5(11): 2981-2988.

[9] Wei, J.F.\& Zhang, Q.\& Zhao, L.\& Kang, W.Y. 2010. Antimicrobial activity of Musa basjoo in vitro. Chin. J. Exp. Tradit. Med. Form. 16: 69-71.

[10] Wang, X.P.\& Xu, S.N.\&Wu, H.M.\& Jin, F.Y. 2011. Study on Chemical Components of Essential Oils from Fresh and Dry Rhizoma Musae by Gas Chromatography-Mass Spectrometry. Chin. J. Exp. Tradit. Med. Form. 17: 82-85.

[11]Xu, F.\& Wu, H.\& Wang, X. P.\& Yang, Y.\& Wang, Y. M.\& Qian, H. B.\& Zhang, Y. Y. 2014. RP-HPLC characterization of lupenone and $\beta$-sitosterol in Rhizoma Musae and evaluation of 
the anti-diabetic activity of lupenone in diabetic sprague-dawley rats. Molecules 19: 1411414127.

[12]Zhang, Q.\& Chang, X.\& Kang, W.Y. 2010. $\alpha$-glucosidase inhibitory activity of Musa Basjoo. Sci. Tech. Food Ind. 31: 125-126, 130.

[13]Zhang, Q.\& Kang, W.Y. 2010. Active constituents of Musa basjoo. Chin. J. Chin. Mater. Med. 35: 2424-2426.

[14]Zhu, S.Q.\& Mo, S.Z. 1989. The alkaloids of Musa basjoo pseudostem on blood pressure in dogs. Mod. Appl. Pharm. 6: 47. 